\title{
Research on the Hybrid Recommendation Method of Retail Electricity Price Package Based on Power User Characteristics and Multi-Attribute Utility in China
}

\author{
Yongxiu He ${ }^{1,2}$, Meiyan Wang ${ }^{1,2, *(\mathbb{D})}$, Jinxiong $\mathrm{Yu}^{1,2}$, Qing $\mathrm{He}^{1,2}$, Huijun Sun ${ }^{1,2}$ and \\ Fengyu Su ${ }^{1,2}$ \\ 1 School of Economics and Management, North China Electric Power University, Beijing 102206, China; \\ yongxiuhe@126.com (Y.H.); jinxiong.yu@163.com (J.Y.); 15650798256@163.com (Q.H.); \\ 15295931235@163.com (H.S.); fuyuso@126.com (F.S.) \\ 2 Beijing Key Laboratory of New Energy and Low-Carbon Development, North China Electric Power \\ University, Beijing 102206, China \\ * Correspondence: meiyan_wang@ncepu.edu.cn; Tel.: +86-61773113
}

Received: 11 April 2020; Accepted: 18 May 2020; Published: 27 May 2020

\begin{abstract}
With the deregulation of the retail electricity market and the increase of the types of electricity price packages, electricity retail companies provide the recommended service of price packages for users, so as to improve the market competitiveness and user stickiness of enterprises. The existing research does not fully consider the impact of user characteristics and package attributes on recommendation results. This paper proposes a hybrid recommendation method of retail electricity price package based on the characteristics of power users and the multi-attribute utility of price package. Firstly, the hierarchical model of hybrid characteristics of power users in retail electricity market is constructed based on the tree structure, and all characteristics are analyzed quantitatively by proximity measurement method. Then, based on the multi-attribute utility theory, the utility model of retail electricity price package to users is constructed. Secondly, the accurate recommendation of the package is realized according to the characteristics of power users and the multi-attribute utility of price package. Finally, the rationality of the hybrid recommendation method of the retail electricity price package is verified by empirical analysis. This study provides valuable support for user to choose the retail electricity price package and improve the competitiveness of power retail companies.
\end{abstract}

Keywords: retail electricity market; the recommendation of retail electricity price packages; characteristics of power users; the multi-attribute utility of price package

\section{Introduction}

Since 2015, with the promulgation of "electric power reform No. 9 document" and supporting documents, the level of China's retail electricity market liberalization has been continuously improved, the retail electricity market users' right of choice has been gradually opened, and diversified power retail competitors have emerged rapidly [1,2]. According to incomplete statistics, by the end of March 2018, there were more than 3342 electricity retail companies registered nationwide [3]. With new participants entering the retail electricity market, the market competition is fierce, and power users have the opportunity to choose from a number of different retailers [4,5]. The relationship between power users and suppliers are being altered [6]. A retail electricity price package is a new type of power product which is developed according to the characteristics of users. Combined with the characteristics of users, a variety of electricity price packages are designed to meet the differentiated needs of user [7]. According to statistics, as of September 2016, there are more than 9000 electricity price packages for users to choose in the end market of Texas, the United States. Through comprehensive evaluation, 
the price comparison website provides users with price package recommendation service $[8,9]$. Since 1998, when the right of choice for users was released in the UK, all users have the freedom to choose electricity suppliers [10]. In the market-oriented transaction, there are many kinds of retail electricity price packages with complex contents, which make it difficult for users to find the most suitable price package for their own electricity demand [11,12].

In view of the large amount of information of user characteristics and the personalized power demand of power users, it is necessary to introduce a recommendation system [13] to help users quickly select suitable retail electricity price packages. A recommendation system is an effective way of information filtering. It can solve the problem of "information overload" and realize professional recommendation service. The core technology of a recommendation system is a recommendation algorithm, which includes content-based recommendations, collaborative filtering recommendations, and hybrid recommendation algorithms [14]. At present, there are some services that directly or indirectly help users choose power products in the power market. Direct methods are popular on online product recommendation systems, such as Power to Choose, Energy Made Easy (EME), Check24, and iSelect [15-18]. The basic principle is to find cheaper power products by directly comparing the costs of all plans. The cost of a product is calculated based on the customer's total electricity usage and the charge rates of the electricity price. Generally, total electricity consumption is estimated based on the corresponding values on the latest electricity bills. A major limitation of the direct manner is that the total power estimate is inaccurate due to neglect many other key factors affecting electricity consumption, such as simultaneity rate and peak valley ratio of customers. Another negative is that these tools don't recognize prices in different periods of the tariff package. Customers have to choose a package with the lowest average electricity price, but it may lead to higher electricity charges in the end. Different from directly comparing costs of all packages, the indirect manner is to recommend electricity packages according to customer characteristics and similar customers' electricity consumption plans $[19,20]$. In order to provide recommendation services to target users, the recommendation system analyzes its energy consumption mode, finds users with similar energy consumption mode from the user knowledge base, and then recommends the most appropriate pricing scheme to the target user [21]. Yuan discuss the scheme recommendation of power price by analyzing the characteristics of power users. According to the data of household appliances obtained by the power plan recommendation system, they can choose the appropriate power scheme for the users [22]. Yang tapped users' potential preferences based on tag collaborative filtering algorithm, and then recommend products in the user's preferences scope. These methods solve the problem of single Recommendation Index of traditional methods [23].

In the above study, the power product recommendation is realized by analyzing the user's power demand preference and historical power use. There is no comprehensive consideration of the attributes of an electricity price package, which affects the accuracy of recommended results. Some studies consider the sensitivity of users to the attributes of an electricity price package. Based on the collaborative filtering algorithm, Shun established a new matrix decomposition algorithm, which can recommend the most appropriate pricing scheme to any target user according to the distribution of electricity consumption and the user's preference for each pricing scheme [24]. Diego proposed a recommendation system that can collect consumption data from electronic products in a home, obtain information on electricity prices in the Internet. Then provide recommendations combine consumption data of users and attributes of price package [25]. The system can optimize the power consumption in different periods of the day, and save users' expenses. The weakness of the existing plan recommendation method is that it overestimates the abilities of the residential user to estimate the price package attributes, e.g., the price of electricity price package in different periods. Due to the lack of accurate evaluation of attributes of the price package, the suggestions provided by these indirect ways are unreliable. On the whole, more and more researches are based on the score of user evaluation to project and complete the recommendation. The existing scholarship in this field, however, tends to overlook the discussion of the characteristics of new users and lacks the recommendation of new 
projects. Another limitation associated with the package recommendation lies with the incomplete description of user characteristics. Lack of unified quantitative analysis of quantitative and qualitative characteristics of users. The sensitivity of users to package utility is ignored, and the multidimensional attribute of package is not considered comprehensively.

Based on the above research background, we proposed a hybrid recommendation method of retail electricity price packages based on the characteristics of power users and multi-attribute utility. On the one hand, the characteristics of power users reflect the current power demand of users [26]. The traditional recommendation algorithm calculates the similarity between users according to the customer's rating of products, so as to realize product recommendation, such as recommendation of movies and books [27-30]. In view of the lack of user rating data, this paper uses a tree structure to describe user characteristics comprehensively. The proximity measure is introduced to quantify the mixed features of the users. By calculating the similarity of user characteristics, the initial recommendation of tariff package is completed. This method does not rely on the scoring data of customer evaluation package, and can solve the problems of data sparsity and cold start in collaborative filtering recommendation method, and can cover users with special interest preferences. On the other hand, considering the sensitivity of users to the utility of electricity price package [31], a collaborative filtering recommendation algorithm based on multi-attribute utility is proposed. The core problem of the algorithm is to build multi-attribute utility function to calculate the comprehensive utility of electricity price package to users [32,33]. Multi attribute utility theory can analyze the utility of a recommendation package to users from the perspective of personalized utility [34,35]. At the same time, non-package attributes, such as vendor reliability and product availability, can be considered in the utility calculation. The algorithm is suitable for the multi-attribute characteristics of power price package. It solves the problem that users can't judge the utility of electricity price package intuitively, and provides the basis for users to choose package. Therefore, combined with the characteristics of power users and multi-attribute utility, the hybrid recommendation can select the most suitable power price package for the target users. At the same time, the hybrid recommendation algorithm presented in this paper is suitable for the recommendation of new projects without scoring data. It is also suitable for recommendation of items with multiple attributes, such as a mobile package recommendation.

The purpose of this study is to design a method for recommendation services and improve the shortcomings of traditional recommendation methods. This paper demonstrates whether the hybrid recommendation method combined with user characteristics and utility of electricity price package is reasonable. The empirical study shows that the recommended electricity price package can significantly reduce the total cost of electricity consumption compared with the current electricity consumption plan of the target users. In addition, we found that considering user characteristics and user preferences for the attributes of electricity price package can avoid the impact of active users and popular packages on the results and increase the coverage of recommendation results.

This study expands research on package recommendation from three main perspectives. Firstly, analyzing the characteristics of power users from three dimensions of basic attributes, consumption attributes and current user's electricity price attributes. The proximity measure is introduced to quantify the different data types of user characteristics. We can find the similar neighbors of the target users more accurately, and determine the initial recommendation set of an electricity price package. Secondly, based on the multi-attribute utility theory, this paper analyzes the comprehensive utility of retail electricity price package to users from the two aspects of electricity expenditure and electricity consumption mode. Finally, based on the power users' similarity matrix and comprehensive utility of electricity price package, the hybrid recommendation model of a price package is constructed by using collaborative filtering and utility recommendation algorithm. This method can recommend projects for new users, and can also recommend new projects to all users. The results of the paper are useful for the companies to provide better recommendation services, guide user to choose a suitable package. To the best of our knowledge, there are few researches on the retail electricity price package recommendation method in China. Therefore, this paper makes a contribution to the research in this field. 
This paper is organized as follows: Section 2, the hierarchical relationship of power user characteristics is established by using a tree structure diagram and discussing user characteristics and building user characteristics matrix. Section 3, the proximity measurement method is used to quantify the user features, and the similarity function of user features is established. Based on the multi-attribute utility theory, the utility function of retail electricity price package to users is constructed. On this basis, the hybrid recommendation model of retail electricity price package is constructed. Section 4 combined with the hybrid recommendation model, taking Tianjin as an example, the paper analyzes the recommended results of electricity price package, and verifies the effectiveness of the model. Section 5 gives conclusion and future works.

\section{Analysis of Power User Characteristics}

This paper analyzes power user characteristics based on the tree structure diagram, and constructs the characteristic matrix of power users.

\subsection{Hierarchical Model of Power User Characteristics}

The electricity price package is formulated by the power retail company according to the characteristics of typical power users. Complete package recommendation based on similarity between target users and typical users. This paper explores the characteristics of power users from three dimensions: the basic attributes of users, the consumption attributes, and the current price attributes of users, and establishes the hierarchical model of power user characteristics by using the tree structure diagram. The data model that uses the tree structure to express the attribute type and the relation between attributes is called the hierarchical model. The data structure of the hierarchical model is simple and provides good logical support. The hierarchical model of user features can describe the connection and constraint relationship between user features more clearly, and extract the information of user feature matrix at the same time. According to the power user feature matrix, the user similarity matrix is obtained. On this basis, the price package recommendation is realized. In Figure 1, the power user acts as the root node, and the basic attribute, the user's consumption attribute and the current user's price attribute are three leaf nodes (i.e., the rounded rectangle in Figure 1). Each leaf node also includes user characteristics. The nodes do not overlap each other.

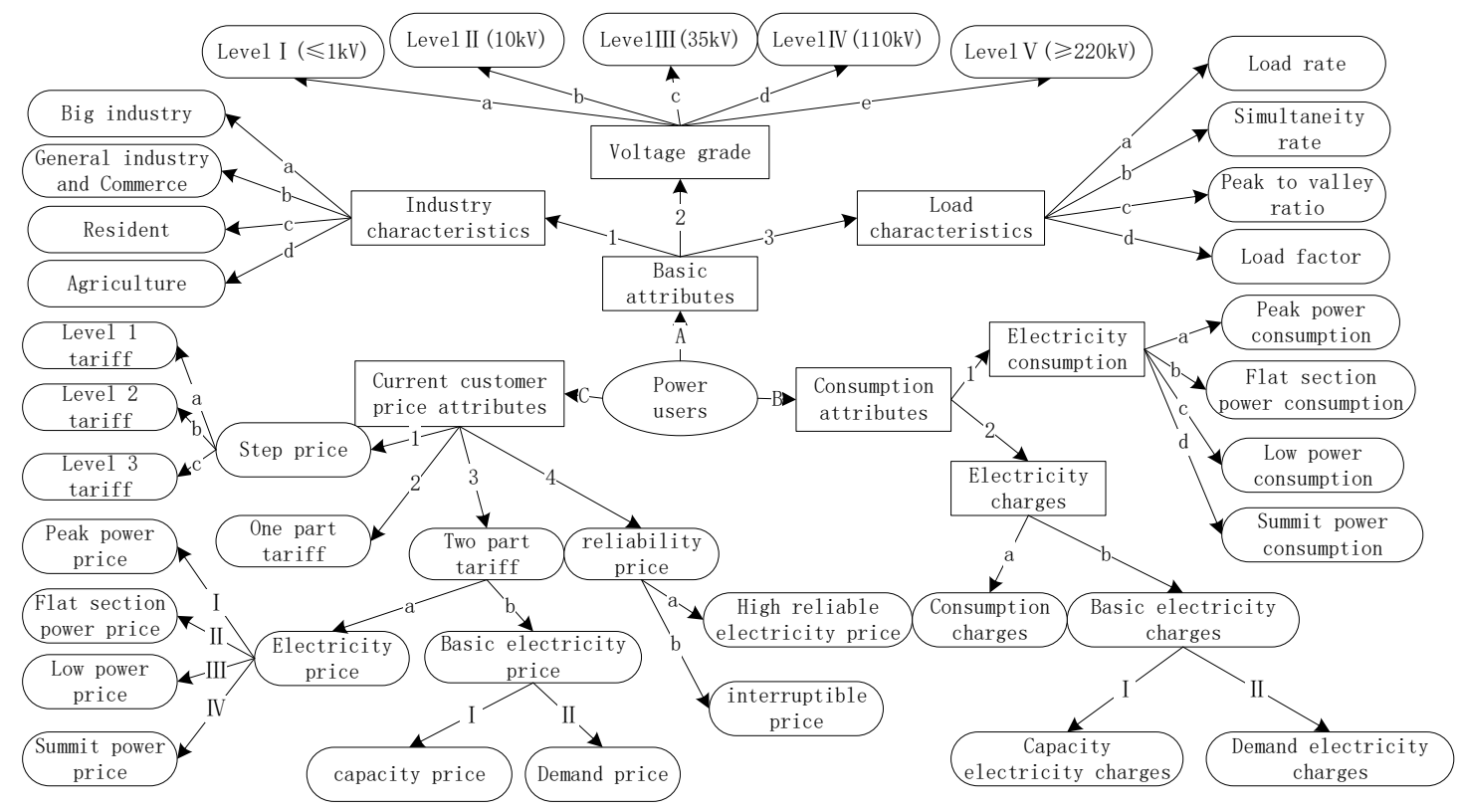

Figure 1. Tree structure chart of power user characteristics. 
In Figure 1, the power users are divided into industry, retail, resident, and agriculture according to the industry characteristics. The voltage level is divided into five levels: no more than $1,10,35$, 110, and no less than $220 \mathrm{kV}$. The load rate, simultaneity rate and peak valley ratio are selected to analyze the load characteristics of power users. Then it analyzes the characteristics of users from the perspective of consumption attributes and current power price attributes of users.

\subsection{Construction of Power User Characteristic Matrix}

According to the results of the power user characteristic analysis, the power user characteristic matrix was constructed. $\left\{u_{1}, u_{2}, u_{3}, \cdots, u_{j}, \cdots, u_{m}\right\}$ represents the collection of $m$ typical users corresponding to the power price package in the system. $\left\{f_{1}, f_{2}, \cdots, f_{z}\right\}$ is the user characteristics 1,2 $\ldots, z$ contained in the system. The user characteristic matrix is expressed as:

$$
F=\left[\begin{array}{cccc}
f_{11} & f_{12} & \cdots & f_{1 z} \\
f_{21} & f_{22} & \cdots & f_{2 z} \\
\vdots & \vdots & \ddots & \vdots \\
f_{m 1} & f_{m 2} & \cdots & f_{m z}
\end{array}\right]
$$

where $f_{m z}$ represents the data of the $z$ - th characteristic of a typical user $u_{m}$.

\section{Hybrid Recommendation Model Based on Power User Characteristics and Multi-Attribute Utility}

The typical user set selected in this paper is the basis for formulating the electricity price package. Each price package corresponds to a typical user. The similarity between the typical user and the target user is calculated, and the similar user set is obtained by ranking the similarity from high to low. The retail electricity price package corresponding to the similar user set constitutes the initial recommendation set of the target user. Then calculate the utility value of the electricity price package to the target user in the initial recommendation, and recommend the retail electricity price package with the largest utility value to the target user.

\subsection{Similarity Analysis of Power Users' Characteristics}

The characteristics of power users include different data types, which to a certain extent determines the preferences of consumers for retail price electricity packages.

According to the proximity measurement methods of different types of data proposed by Han, the attributes of power user characteristic data are divided into nominal type, numerical type, and ordinal type, as shown in Table 1.

Table 1. Characteristic types of power users.

\begin{tabular}{|c|c|c|}
\hline Feature Types & Feature & Power User Characteristic Attribute \\
\hline Nominal type & $\begin{array}{l}\text { The user's characteristic is the name of the } \\
\text { object }\end{array}$ & Industry, Retail, Resident and Agriculture \\
\hline Numerical type & Expressed as an integer or real value & $\begin{array}{l}\text { Load rate, Simultaneity rate and Peak to } \\
\text { valley ratio }\end{array}$ \\
\hline Ordinal type & $\begin{array}{l}\text { Meaningful ordering or ranking between } \\
\text { ordinal attribute values }\end{array}$ & The voltage level \\
\hline
\end{tabular}

It can be seen from Table 1 that the characteristic attributes of power users belong to a mixed type, and the proximity measurement method is applied to analyze the mixed characteristics of the power users. Assuming there are $n$ target users in total, which is represented by the set $U=\left\{u_{1}, u_{2}, \cdots, u_{n}\right\}$, where $u_{i}$ is the $i-$ th target user. Take $m$ typical users corresponding to the recommended price package of the electricity selling company, which is represented by the set $R=\left\{r_{1}, r_{2}, \cdots, r_{m}\right\}$. Where $r_{j}$ 
represents the typical users corresponding to the $j-$ th package. The dissimilarity between the typical user $r_{j}$ and the target user $u_{i}$ is calculated by the following formula.

$$
d\left(u_{i}, r_{j}\right)=\frac{\sum_{f=1}^{z} \partial_{u_{i}, r_{j}}^{(f)} d_{u_{i}, r_{j}}^{(f)}}{\sum_{f=1}^{z} \partial_{u_{i}, r_{j}}^{(f)}}
$$

where $d\left(u_{i}, r_{j}\right)$ represents the difference between the typical user $r_{j}$ and the target user $u_{i} ; f$ represents the power user characteristics; $z$ represents the number of power user characteristics; $\partial_{u_{i}, r_{j}}^{(f)}$ is the indicator; $d_{u_{i}, r_{j}}^{(f)}$ is the contribution of feature $f$ to the dissimilarity between users $u_{i}$ and $r_{j}$.

The fetching value principle of the $\partial_{u_{i}, r_{j}}^{(f)}$ : If $x_{u_{i} f}$ or $x_{r_{j} f}$ is missing (That is, the target user $u_{i}$ or typical user $r_{j}$ does not have a measure value of characteristic $f$ ), or $x_{u_{i} f}=x_{r_{j} f}=0$, and $f$ is an asymmetric binary user feature, then $\partial_{u_{i}, r_{j}}^{(f)}=0$; otherwise $\partial_{u_{i}, r_{j}}^{(f)}=1$.

The fetching value principle of the $d_{u_{i}, r_{j}}^{(f)}$ :

(1) If the characteristic $f$ of power users is numerical type, then $d_{u_{i}, r_{j}}^{(f)}=\frac{\left|x_{u_{i f}}-x_{r_{j} f}\right|}{\left(\max _{h} x_{h f}\right)}$, where $h$ can take all the non-missing value of the characteristics $f$ of the power user.

(2) If the characteristic $f$ of power users is nominal type or binary type, when the characteristic value $x_{u_{i} f}=x_{r_{j} f}$, then $d_{u_{i}, r_{j}}^{(f)}=0$; otherwise, $d_{u_{i}, r_{j}}^{(f)}=1$.

(3) If the characteristics $f$ of power users are ordinal: calculate ranking $r_{u f}$ and $Z_{u f}=\frac{\left(r_{u f}-1\right)}{\left(m_{f}-1\right)}$, where, $r_{u f}$ represents the ranking of state in the characteristic $f$ of power users; $m_{f}$ is the number of ordered states of $f$, and treat $Z_{u f}$ as a numerical type.

Calculate the similarity between target users $u_{i}$ and typical users $r_{j}$ :

$$
\operatorname{sim}_{f}\left(u_{i}, r_{j}\right)=1-\frac{\sum_{f=1}^{z} \partial_{u_{i}, r_{j}}^{(f)} d_{u_{i}, r_{j}}^{(f)}}{\sum_{f=1}^{z} \partial_{u_{i}, r_{j}}^{(f)}}
$$

where $\operatorname{sim}_{f}\left(u_{i}, r_{j}\right)$ is feature similarity of power users.

Building user feature similarity matrix based on user feature similarity calculation results, which is a matrix of $m \times m$ order and represented by $M$ :

$$
M=\left[\begin{array}{cccc}
1 & s_{12} & \cdots & s_{1 m} \\
s_{21} & 1 & \cdots & s_{2 m} \\
\vdots & \vdots & \ddots & \vdots \\
s_{m 1} & s_{m 2} & \cdots & 1
\end{array}\right]
$$

where $s_{i j}$ is the feature similarity value between target user $u_{i}$ and typical user $r_{j}$.

\subsection{Multi Attribute Utility Function of Electricity Price Package}

When users purchase the electricity price package, they tend to choose the package with greater utility. In this paper, multi-attribute utility theory is applied to evaluate the utility of electricity price package attributes to users, providing scientific basis for users to purchase package. This paper explores the impact of the attributes of the electricity price package to the utility of the target user from the two aspects of electricity expenditure and electricity consumption mode. 
Assuming that each electricity price package has $m$ attributes, $x_{k}$ indicates the value of the $k-$ th attribute of the electricity price package. Then the electricity price package $P$ can be expressed as a vector composed of $m$ attribute values $P=\left(x_{1}, x_{2}, \ldots, x_{m}\right)$.

Let $x_{k}$ have $l_{k}$ discretized attribute values, which form the value set of attribute $k$ :

$$
V_{k}=a_{k, 1}, \ldots, a_{k, s}, \ldots, a_{k, l_{k}}
$$

where $a_{k, s}$ represents the $s-$ th value of attribute $k$. The attribute $k$ of the electricity price package can only take one value.

(1) Utility of Attribute Value of Electricity Price Level to Users

Considering the different price levels of the electricity price package during each period, the peak and valley power consumption of users in different seasons is first predicted, and calculate the monthly average power consumption. Then, the user's electricity consumption fee is estimated based on the price level of the current electricity consumption plan and the predicted power consumption. The formula is:

$$
\mathrm{R}_{\mathrm{i}, \mathrm{j}}=\sum_{\mathrm{t}=1}^{\mathrm{T}}\left(\mathrm{q}_{\mathrm{it}} \times \mathrm{P}_{\mathrm{jt}}\right)+\mathrm{P}_{\mathrm{cj}}(i=1,2, \cdots, \mathrm{I} ; j=1,2, \cdots, \mathrm{J})
$$

where $R_{i j}$ is the consumption cost of the $i$ - th target user if they choose the $j$ - th electricity price package; $q_{i t}$ is the estimated power usage of the $i$ - th target user for the $t$ - th time period; $P_{j t}$ and $P_{c j}$ represent, respectively, the power energy price and the capacity price of the $j-$ th price package for the $t$ - th time period. I represents the total number of users, $J$ represents the total number of retail power price packages, and $T$ is the number of time periods.

On the other hand, for the $i-$ th target user, before buying a certain retail electricity price package, there will be a basic electricity consumption budget, which is represented by $B_{i}(i=1,2, \cdots, I)$. The difference between $B_{i}$ and $R_{i j}$ represents the benefits of the $j$ - th electricity price package. The greater the difference, the higher the purchase intention of users. The formula is as follows:

$$
u_{i j}=B_{i}-R_{i j}(i=1,2, \cdots I ; j=1,2, \cdots J)
$$

where $u_{i j}$ is the benefit of the $i$ - th target user choosing the $j$ - th electricity price package.

(2) The Utility of Structure Attribute of Retail Electricity Price Package to Users

Structure attribute is the key factor that affects the utility of electricity price package to users. The peak and valley period of price package affects the way users use electricity.

Before implementing the retail electricity price package, the user does not have a response because of the same price of each period, so the user will choose the most comfortable way to use electricity, which usually acts as the reference of the user's satisfaction of electricity consumption mode. After the implementation of the electricity price package, the structure attribute of the price package causes the change of the power consumption mode of the users and forms a new load curve. The difference value between the electricity quantity and the reference in each period after the implementation of the tariff package reflects the applicability of the package. The smaller of the difference value, the more willing users are to buy. The formula is as follows:

$$
u_{i j}^{\prime}=1-\frac{\sum_{t-1}^{24}\left|Q_{t}^{\prime}-Q_{t}\right|}{\sum_{t-1}^{24} Q_{t}}
$$

where $u_{i j}^{\prime}$ represents the utility of retail electricity price package structure attribute to users; $Q_{t}$ is the power consumption of $t$ period before the implementation of the electricity price package; $Q_{t}^{\prime}$ represents the power consumption of period $t$ after the implementation of the retail electricity price package.

(3) Comprehensive Utility of the Electricity Price Package to Users 
The comprehensive utility of the electricity price package to users is the weighted average of the utility of electricity price level and structure attribute to users. The formula is as follows:

$$
U_{i j}=w_{1} u_{i j}+w_{2} u_{i j}^{\prime}
$$

where $U_{i j}$ represents the comprehensive utility of retail electricity price package to users. $w_{1}$ represents the proportion of utility generated by electricity price level of retail electricity price package to comprehensive utility. $w_{2}$ represents the proportion of utility generated by the structural attribute of retail electricity price package to the comprehensive utility.

The weight is determined according to the number of times when users browse the attributes of electricity price package. The price package contains the attribute $k$ and attribute value $a_{k, s}$ of user preference. If the attribute value $a_{k, s}$ of attribute $k$ appears more times, the weight of attribute $k$ is greater. For example, the user's browsing records are all about the price level in peak period, which shows that the price level has a great impact on the user's utility, so the price level should be given a greater weight.

Suppose user $i$ has clicked to view $n$ retail electricity price packages before making a purchase plan. Attribute value a of attribute $k$ appears $f_{i, k, s}$ times. If $\max \left(f_{i, k, s}\right)$ deviates from the average frequency $n / l_{k}$ and is closer to $n$, the greater the impact of attributes on user utility, the greater the weight of attribute $k$ should be given, otherwise, the weight of attribute $k$ is smaller. The weight formula of the attribute is:

$$
w_{i, k}=\left[\max \left(f_{i, k, s}\right)-\frac{n}{l_{k}}\right] / \sum_{k=1}^{m}\left[\max \left(f_{i, k, s}\right)-\frac{n}{l_{k}}\right]
$$

where $w_{i, k}$ is the weight that affects utility; $f_{i, k, s}$ represents the number of times that attribute value $a_{k, s}$ of attribute $k$ appears; $n / l_{k}$ is the average number of occurrence of attribute values; $m$ represents the sums of electricity price package attributes.

\subsection{Hybrid Recommendation Algorithm Based on User Characteristics and Electricity Price Package Utility}

The recommendation is completed by analyzing the characteristics of power users and the utility of retail electricity price package. First, the collaborative filtering recommendation is applied to get the initial set of retail electricity price package recommendations, and then the utility recommendation means is applied to get the final list of recommendations for the target users. The specific steps are as follows:

Input: characteristic matrix $F$ of power users, attribute value of retail price package.

Output: Retail electricity price package recommendation set $\operatorname{Rec}$ of target user $u_{i}$.

Step 1: Explore the characteristics of power users and build the user characteristic matrix $F$.

Step 2: Based on the user's characteristic matrix $F$, the feature similarity $\operatorname{sim}_{f}\left(u_{i}, r_{j}\right)$ between the typical user $r_{j}$ and the target user $u_{i}$ is calculated according to formula (3), and the user's feature similarity matrix $M$ is obtained.

Step 3: According to the value of similarity $\operatorname{sim}_{f}\left(u_{i}, r_{j}\right)$, the typical users are sorted, and the typical users with high similarity are selected to form a similar user set, which is represented by $N_{u_{i}}$.

Step 4: Find the code of the retail price package corresponding to $N_{u_{i}}$, and eliminate the duplicate code, that is, get the initial retail price package recommendation set $T_{u}$ of user $u_{i}$.

Step 5: According to the multi-attribute utility model, the utility of the initial electricity price package to users is calculated. The utility value is sorted from high to low and the recommendation list Top $-N$ of the target user is obtained to recommend to the user.

This paper presents a flow chart of hybrid recommendation algorithm based on the characteristics of power users and the utility of retail power price package, as shown in Figure 2. 


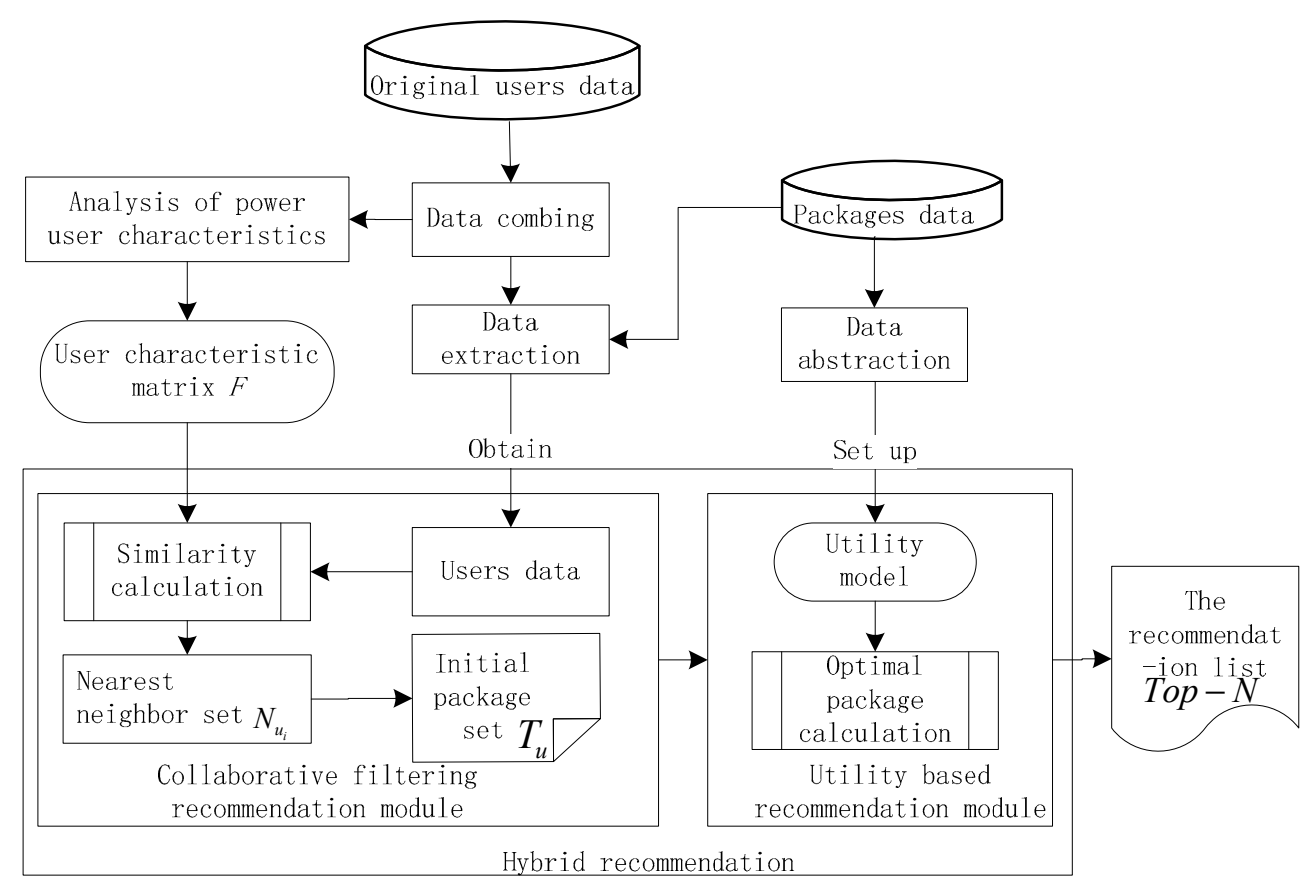

Figure 2. The step of the hybrid recommendation method.

\section{Case Analysis}

In this section, the initial recommended electricity price package set and the final package recommendation results are given. The accuracy and coverage are used to evaluate the rationality of the hybrid recommendation method. In the case study, we recommend the electricity price package with the maximum utility value for users, and the total electricity cost is the lowest when the package is implemented.

\subsection{Basic Data}

Taking Tianjin large industry as a case, the load characteristic data of 50 typical users are provided by State Grid Tianjin electric power company. User voltage types include: 10, 35, 110, and $220 \mathrm{kV}$. An example of user characteristic data is shown in Table 2.

Table 2. User characteristic data.

\begin{tabular}{ccccccc}
\hline User & $\begin{array}{c}\text { Industry } \\
\text { Characteristics }\end{array}$ & $\begin{array}{c}\text { Voltage } \\
\text { Grade/kV }\end{array}$ & $\begin{array}{c}\text { Monthly } \\
\text { Load Rate }\end{array}$ & $\begin{array}{c}\text { Simultaneity } \\
\text { Rate }\end{array}$ & $\begin{array}{c}\text { Peak to } \\
\text { Valley Ratio }\end{array}$ & $\begin{array}{c}\text { Monthly } \\
\text { Electricity } \\
\text { Consumption } \\
\text { /GWh }\end{array}$ \\
\hline$u_{1}$ & large industrial & 10 & 0.652 & 0.86 & 1.245 & 332.643 \\
$u_{2}$ & large industrial & 10 & 0.553 & 0.83 & 1.126 & 390.369 \\
$u_{3}$ & large industrial & 35 & 0.437 & 0.88 & 1.102 & 205.378 \\
$u_{4}$ & large industrial & 35 & 0.702 & 0.91 & 1.080 & 369.259 \\
$u_{5}$ & large industrial & 110 & 0.741 & 0.94 & 1.021 & 385.131 \\
\hline
\end{tabular}

The attributes of retail electricity price package include: capacity price and price in peak and valley periods of different seasons. As illustrated in Table 3. 
Table 3. Alternative scheme of electricity price package for large industry.

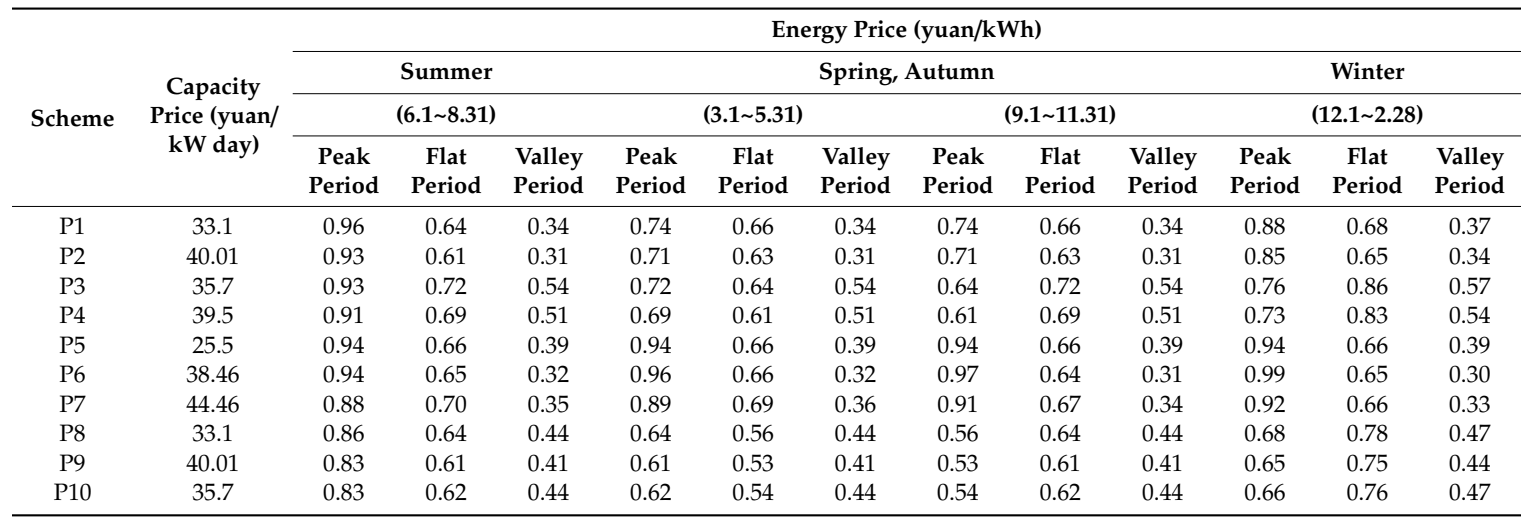

\subsection{Analysis on the Recommendation Results of Retail Electricity Price Package}

\subsubsection{Initial Recommendation Results of Retail Electricity Price Package Based on User Characteristics}

The industry category is the nominal attribute. In this paper, the recommended object of retail electricity price package is the large industry, so the industry category similarity of users is 1 . The voltage level is an ordinal attribute. According to a certain order, the voltage level is divided into five levels, and respectively assignment the values are 1,2,3,4, and 5. The value is assigned according to the user's voltage level, and then the voltage dissimilarity is calculated according to the formula of numerical attribute dissimilarity. Monthly load rate, simultaneity rate, peak valley ratio, and monthly average power consumption are all numerical attributes, and the results are obtained according to the formula of numerical attribute dissimilarity. According to the user characteristic data in Table 1, calculate the user's dissimilarity, and convert the dissimilarity into similarity according to Equation (3). The result as shown in Table 4.

Table 4. User similarity measurement results.

\begin{tabular}{cccccccc}
\hline Order Number & $\mathbf{1}$ & $\mathbf{2}$ & $\mathbf{3}$ & $\mathbf{4}$ & $\ldots$ & $\mathbf{4 9}$ & $\mathbf{5 0}$ \\
\hline 1 & 1 & & & & & & \\
2 & 0.8655 & 1 & & & & & \\
3 & 0.9313 & 0.9343 & 1 & & & & \\
4 & 0.9269 & 0.9109 & 0.8800 & 1 & & & \\
$\ldots$ & $\ldots$ & $\ldots$ & $\ldots$ & $\ldots$ & $\ldots$ & & \\
49 & 0.8217 & 0.9171 & 0.8837 & 0.8670 & $\ldots$ & 1 & \\
50 & 0.3739 & 0.2983 & 0.3615 & 0.3504 & 0.4719 & $\ldots$ & 1 \\
\hline
\end{tabular}

In Table 4 , the similarity results on both sides of the diagonal are symmetrical. In order to be easy to understand, only the similarity calculation results of two users below the diagonal are listed. The value 1 on the diagonal represents the similarity between users and themselves.

According to the similarity calculation results in Table 4, we rank the similarity from high to low and find the neighbor set with the highest similarity with the target user. The retail electricity price package selected by similar users is taken as the initial recommendation set of target users, as shown in Table 5 .

Table 5 shows the five typical users that are most similar to the target users, and the electricity price package corresponding to the typical users. The similarity between the typical user and the target user 3 is the highest, which is 0.9313 . Therefore, the price package P6 corresponding to the typical user 3 is the preferred package for the target user. 
Table 5. Retail electricity price package initial recommendation set.

\begin{tabular}{ccccc}
\hline Order Number & Target User ID & Nearest Neighbor ID & Similarity Degree & $\begin{array}{c}\text { Corresponding Retail } \\
\text { Electricity Price Package }\end{array}$ \\
\hline 1 & 1 & 3 & 0.9313 & P6 \\
2 & 1 & 10 & 0.9269 & P1 \\
3 & 1 & 26 & 0.9021 & P3 \\
4 & 1 & 11 & 0.8940 & P7 \\
5 & 1 & 34 & 0.8865 & P5 \\
\hline
\end{tabular}

4.2.2. Analysis of Final Recommendation Results Based on the Utility of the Electricity Price Package

According to the data in Table 3, the attributes of electricity price package include: capacity price, peak time price, valley time price, etc. Each attribute includes ten attribute values. According to the utility calculation steps, the utility of the initial recommendation package to the target user is obtained. Calculate the utility value of P6, P1, P3, P7, and P5 to the target users according to Equations (5)-(9).

The utility value of the price level and structure attribute of the retail electricity price package to the target user is analyzed. Taking the P6 of the retail electricity price package as an example, the calculation results are as follows:

$$
\begin{gathered}
R_{i j}=\sum_{t=1}^{T}\left(q_{i t} \times P_{j t}\right)+P_{c j}= \\
u_{i j}=B_{i}-R_{i j}=45-38.25=6.75 \\
u_{i j}^{\prime}=1-\frac{\sum_{k-1}^{24}\left|Q_{k}^{\prime}-Q_{k}\right|}{\sum_{k-1}^{24} Q_{k}}=0.747
\end{gathered}
$$

The occurrence times of attribute values of retail electricity price package are counted, and the deviation degree between the maximum occurrence times of attribute values and the average frequency is analyzed. In this paper, the weight is determined by the similarity of attribute values between the price package and the current electricity consumption plan of the target user. By comparing the attribute values of the electricity price package and the target user's power consumption plan, the influence weight of the electricity price level on the comprehensive utility is obtained. The power consumption before and after the implementation of the tariff package is compared, and the influence weight of the structure attribute of the tariff package on the comprehensive utility is obtained.

$$
\begin{aligned}
& w_{i, 1}=\left[\max \left(f_{i, k, s}\right)-\frac{n}{l_{k}}\right] / \sum_{k=1}^{m}\left[\max \left(f_{i, k, s}\right)-\frac{n}{l_{k}}\right]=0.796 \\
& w_{i, 2}=\left[\max \left(f_{i, k, s}\right)-\frac{n}{l_{k}}\right] / \sum_{k=1}^{m}\left[\max \left(f_{i, k, s}\right)-\frac{n}{l_{k}}\right]=0.204
\end{aligned}
$$

The comprehensive utility of retail electricity price package to target users is the weighted average of the utility of electricity price level and structure attribute to users. The comprehensive utility of retail electricity price package P6 to users is as follows:

$$
U_{i}=w_{i 1} u_{i 1}+w_{i 2} u_{i 2}=6.75 \times 0.796+0.85 \times 0.204=5.525
$$

Similarly, the utility value of other electricity price packages to target users can be obtained. On the basis of the utility of the retail electricity price package to the target users, the final recommendation list is given, as illustrated in Table 6 . 
Table 6. Recommended results of electricity price package.

\begin{tabular}{cccccc}
\hline $\begin{array}{c}\text { Target } \\
\text { User ID }\end{array}$ & $\begin{array}{c}\text { Nearest } \\
\text { Neighbor ID }\end{array}$ & $\begin{array}{c}\text { Similarity } \\
\text { Degree }\end{array}$ & $\begin{array}{c}\text { Corresponding Retail } \\
\text { Electricity Price Package }\end{array}$ & Utility & Sorting \\
\hline 1 & 10 & 0.9269 & P1 & 6.672 & 1 \\
1 & 3 & 0.9313 & P6 & 5.525 & 2 \\
1 & 11 & 0.8940 & P7 & 4.679 & 3 \\
1 & 34 & 0.8865 & P5 & 3.431 & 4 \\
1 & 26 & 0.9021 & P3 & 2.49 & 5 \\
\hline
\end{tabular}

Table 6 shows the recommended results of electricity price package based on utility value. Combined with the initial recommendation results in Table 5, the utility value of P1 is the highest, which is 6.672. The second is P6, the utility is 5.525. Therefore, the preferred package recommended to the target user is P1.

Based on the above analysis results, this paper compares the current electricity consumption plan of target users with the five recommended price packages. The cost of target users in one day under the six schemes is shown in Table 7 and Figure 3.

Table 7. Cost comparison between current electricity price plan and electricity price package.

\begin{tabular}{ccccccc}
\hline Plan & Current Plan & P1 & P3 & P5 & P6 & P7 \\
\hline $\begin{array}{c}\text { Total cost } \\
\text { (yuan) }\end{array}$ & 200030.8 & 188338.3 & 204110.9 & 205334.8 & 197095.5 & 195211.4 \\
\hline
\end{tabular}

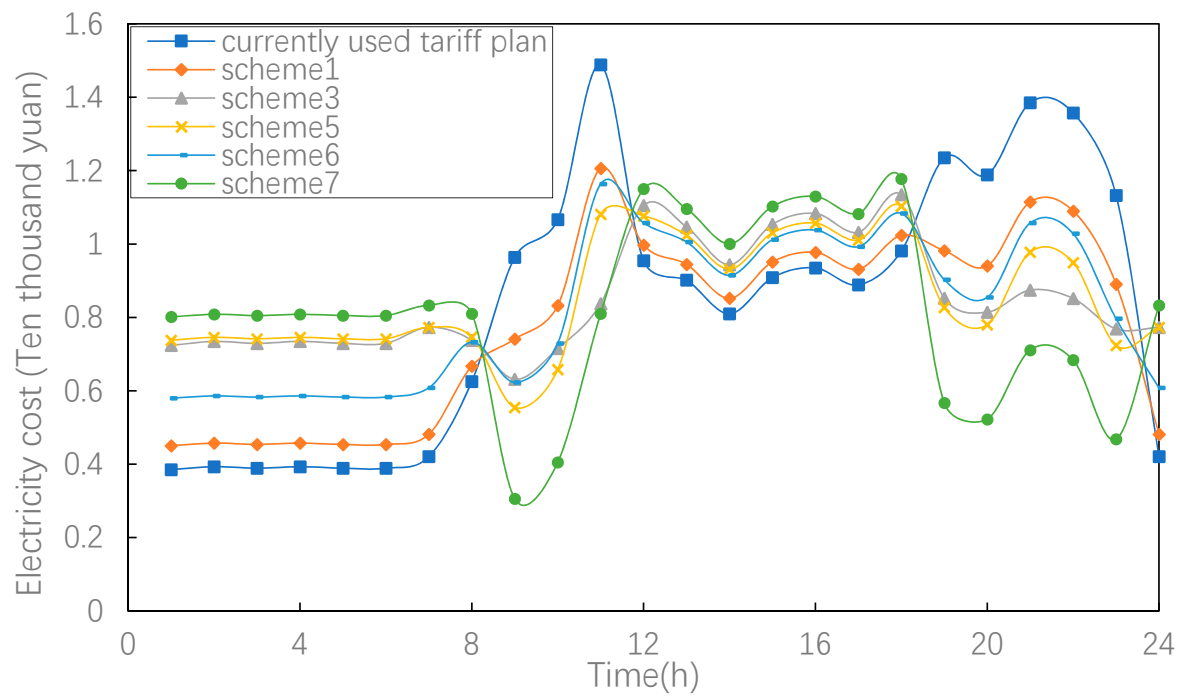

Figure 3. Cost of different price packages used by target users.

In Table 7, the total cost of P1, P6, and P7 is lower than the current electricity price plan, and P1 has the lowest total cost. Consistent with the recommendation results in Table 6, it is determined again that $\mathrm{P} 1$ is the preferred package recommended to users.

In Figure 3, the cost of current electricity price of users in peak hours is higher than the five electricity price packages recommended in this paper. This is because the electricity price in the current peak period is higher than the price package P1, P3, and P7. Moreover, the price difference between peak and valley of the current electricity consumption plan is significantly lower than the price package P5 and P6.

In the vicinity of peak hours, the price package cost recommended by the system is higher than the cost of current electricity consumption plan. This means that the implementation of the tariff package will shift the power consumption during peak hours to other times when the power 
consumption of users is lower. Therefore, when users change the current electricity consumption plan to the recommended tariff package, the peak load of the system may be transferred to other periods. To achieve peak cutting and valley filling, further improve the overall operating efficiency of the power system and optimize the allocation of power resources.

\subsection{Verification of Recommended Model of Retail Electricity Price Package}

In this paper, accuracy and coverage are applied to confirm the rationality of the similarity calculation method and the hybrid recommendation method of electricity price package.

\subsubsection{Evaluating Indicator}

Suppose that the total number of power price packages recommended to user $u$ is $R(u)$, and the set of packages recommended to target user in training set is $T(u)$. Select the accuracy and coverage rate to evaluate the methods proposed in this paper.

(1) The accuracy is expressed as the proportion of forecasting the correct electricity price package in all packages. The higher the accuracy, the better the recommendation result of the electricity price package. The formula of accuracy is:

$$
\text { Accuracy }=\frac{\sum_{u \in U^{\prime}}|R(u) \cap T(u)|}{\sum_{u \in U^{\prime}}|R(u)|}
$$

where $R(u)$ is the set of packages recommended by the recommendation system for the target user $u$, and $T(u)$ is the set of packages recommended to the target user $u$ according to the training set data. The project set of this study refers to the electricity price package.

(2) Coverage indicates the proportion of electricity price packages recommended by the system to users in all retail electricity price packages. The corresponding formula is:

$$
\text { Coverage }=\frac{\left|\sum_{u \in U^{\prime}} R(u)\right|}{I}
$$

where $U^{\prime}$ represents all users, $R(u)$ represents the total number of retail power price packages recommended to users $u$ and $I$ represents the total number of electricity price packages in the system.

\subsubsection{Comparative Analysis of Similarity Calculation Methods}

In order to verify the rationality of the similarity calculation method of hybrid feature attributes of power users proposed in this study, we choose the Pearson correlation coefficient and the Euclidean distance similarity calculation method for comparative experiments. The experimental results were analyzed by using the evaluation indexes of accuracy and coverage. In the test, the number of similar neighbors of the target user is set to $5,10,20,40,60,80$, and 100 and the calculation results are shown in Figures 4 and 5.

As can be seen from the above figure, the proposed similarity calculation method of mixed feature attributes is much more accurate than the traditional similarity algorithm. This is due to the application of proximity measurement method to quantify the hybrid characteristics of power users, which can better explore the similarity between users and improve the accuracy of recommendation.

As can be seen from the above figure, our proposed similarity calculation means has higher coverage than the traditional similarity calculation method. On the three datasets, the coverage decreases with the increase of $k$ value. With the increase of similar neighbors, the system is more inclined to recommend the hot price package, and the recommendation of long tail package is less and less, which leads to the decrease of coverage. 


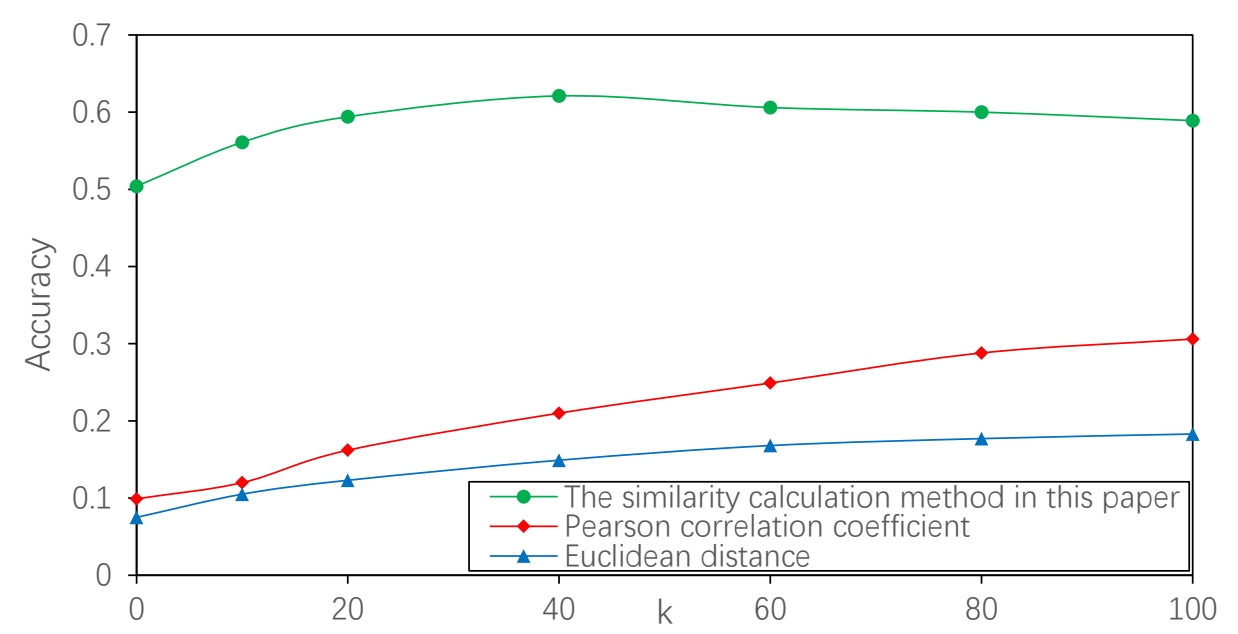

Figure 4. Accuracy of different similarity calculation methods.

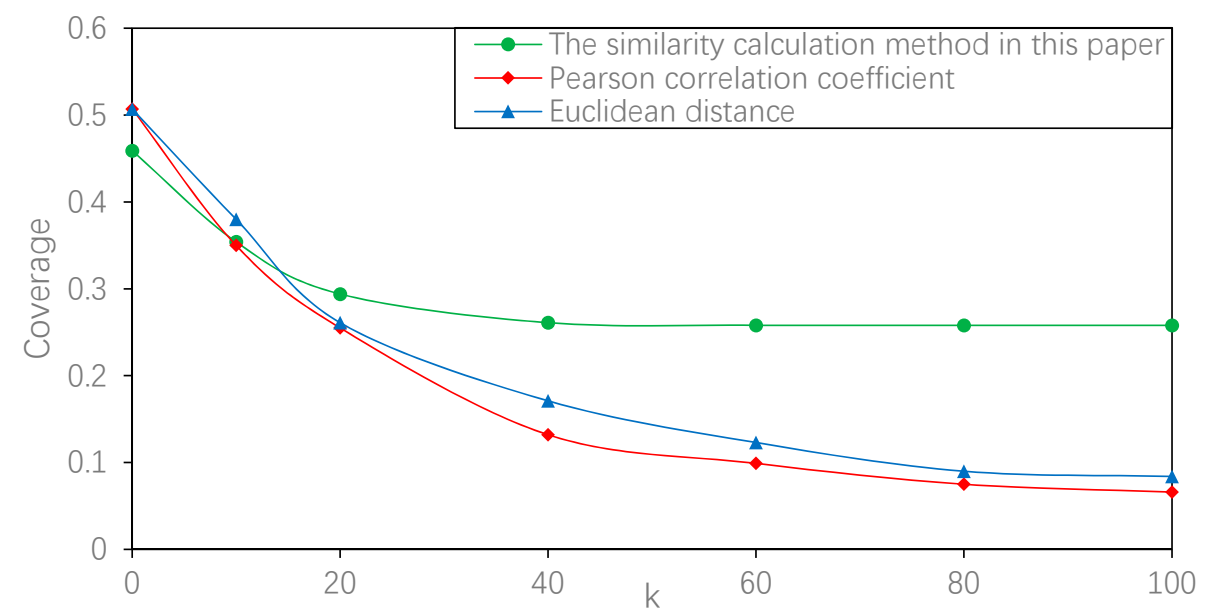

Figure 5. Coverage of different similarity calculation methods.

4.3.3. Validation of Hybrid Recommendation Method for Electricity Price Package

(1) The Accuracy of the Hybrid Recommendation Model of Retail Electricity Price Package

In order to verify the accuracy of algorithm in this paper, four representative recommendation methods are selected for comparative experiments, including slope one, singular value decomposition SVD, collaborative filtering recommendation algorithm based on commodity attribute value (CAU-CF), and traditional collaborative filtering recommendation algorithm (T-CF).

During the experiment, the number of recommended items started from two, and after five, five recommended items were added each time. The experimental results of accuracy are shown in Figure 6.

From Figure 6, it can be seen that compared to other methods, the algorithm adopted in this paper can lead to higher accuracy of retail electricity price package recommendation.

(2) The Coverage of Hybrid Recommendation Model of Retail Electricity Price Package

In the data set, $80 \%$ of users are randomly selected as training data and $20 \%$ as test data. For the setting of the value of user's nearest neighbor $k$, the value is between [10,100]. The coverage of algorithm in this paper, user-based collaborative filtering (UB-CF) and multi-attribute utility collaborative filtering (MAU-CF) are compared and analyzed. The results are shown in Figure 7. 


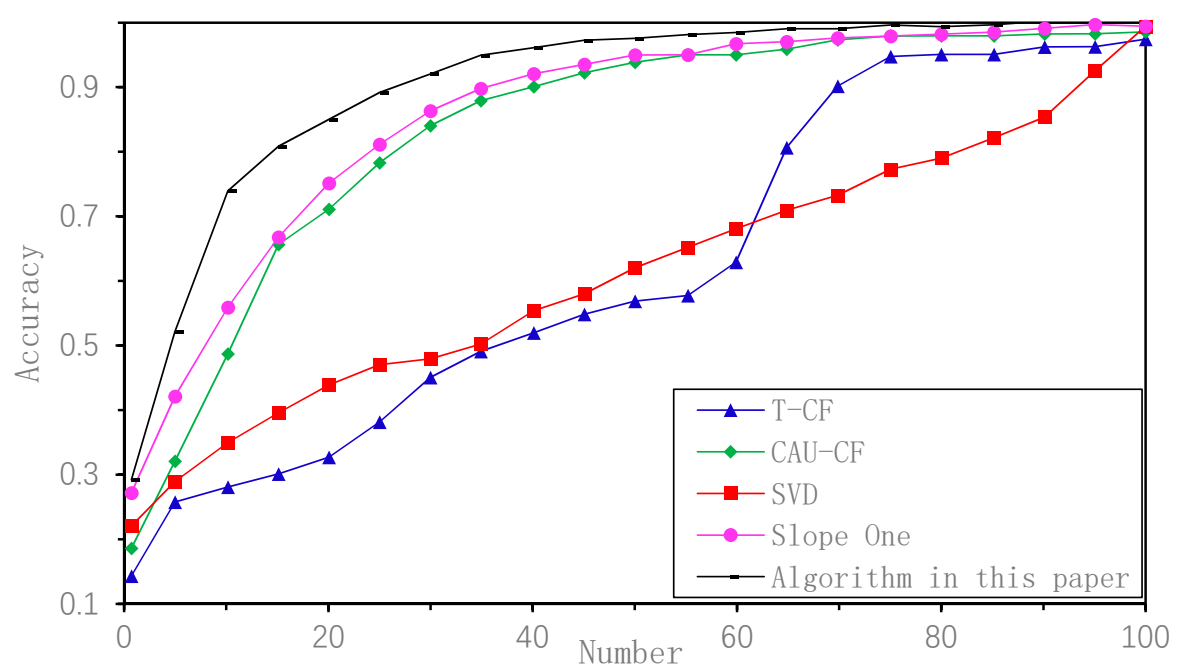

Figure 6. The accuracy of various algorithms.

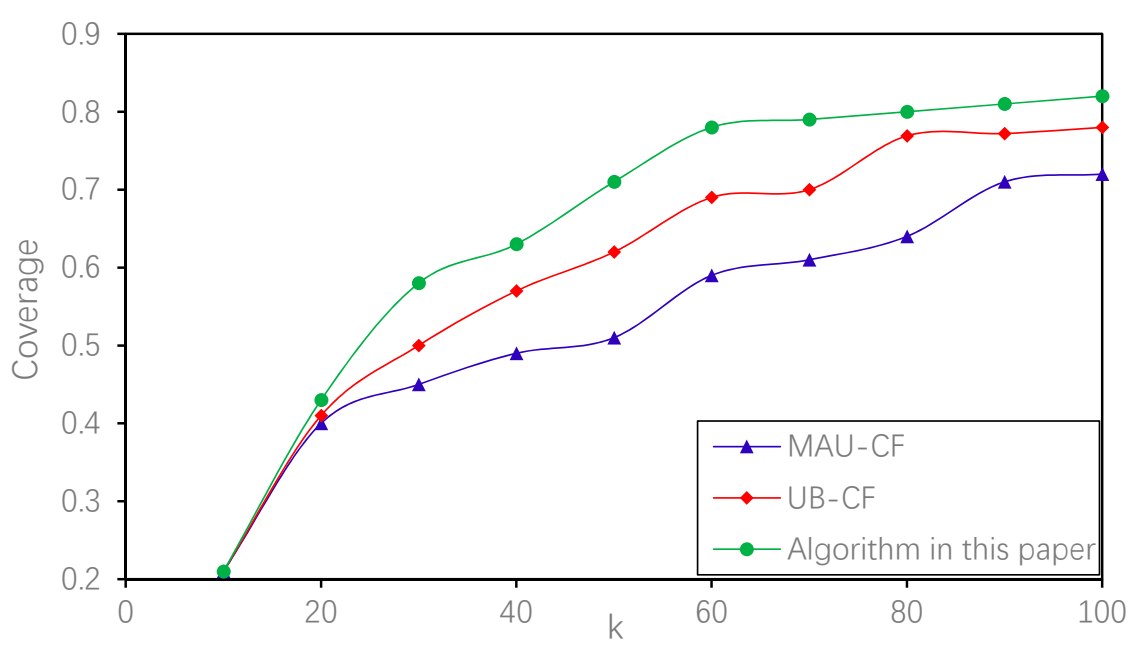

Figure 7. The coverage of various algorithms.

In Figure 7 , in the $k$ value range of $[10,100]$, the algorithm in this paper improves the coverage of the package. The coverage rate fluctuates with the change of $k$ value. Whether it is the maximum fitness or the minimum, the algorithm in this paper can obtain the best coverage rate. This is because the hybrid recommendation algorithm proposed in this paper considers the utility of electricity price package to users. According to users' preference for package attributes, package recommendation can avoid the impact of active users and popular packages. With the increase of the number of similar neighbors, the recommendation results will be more diverse and the coverage will be higher.

\section{Conclusions}

This paper aims at the situation that the power consumers freely choose the electricity retail companies after the opening of the retail electricity market. We propose a method to realize the recommended service of electricity price package for the electricity retail company. That is, the hybrid recommendation method combining the user characteristics and the utility of price package. The conclusions of this study are as follows:

(1) The hierarchical model of power user characteristics is built based on a tree structure chart to analyze the characteristics of different power users. According to the analysis results, the electricity retail company can provide retail electricity price package recommendation services for 
different types of users, so as to increase the viscosity of users and improve the competitiveness of electricity retail enterprises.

(2) In this paper, proximity measurement is used to quantify the quantitative and qualitative characteristics of power users. At the same time, based on the multi-attribute utility theory, the utility of retail electricity price package to users is analyzed from two aspects of electricity expenditure and electricity consumption mode. The two methods provide conditions for the study of hybrid feature similarity and multi-attribute utility of retail electricity price package.

(3) This paper proposes a hybrid recommendation method based on the characteristics of power users and the utility of retail electricity price packages. First of all, according to the similarity matrix of user characteristics, the neighbor user set of the target user is obtained, and the initial retail electricity price package recommendation set of the target user is obtained based on the collaborative filtering recommendation algorithm. Then, based on the utility recommendation algorithm, the utility of electricity price package in the initial recommendation set to the users is calculated, and the final recommendation list of the target users is obtained.

(4) In this paper, accuracy and coverage are used to verify the effectiveness of the retail electricity price package recommendation model. The study results show that the proposed algorithm has a higher success rate than the traditional collaborative filtering algorithm and utility recommendation algorithm, and is more suitable for the electricity retail companies to provide the recommended service of tariff package for customers.

However, the hybrid recommendation method has only been verified in a theoretical case studies and not in realistic environment. This will be one aspect of our future research.

In the future, with the gradual opening of the retail electricity market, the characteristics of power users will be more prominent, and the attributes of the retail electricity price package set by the electricity retail companies will be more diversified. Therefore, we can apply the multi-attribute utility theory to study the new attributes of retail electricity price package, the reliability of electricity retail companies and the availability of power products.

Author Contributions: Conceptualization, Y.H.; methodology, M.W.; software, M.W.; validation, M.W. and J.Y.; formal analysis, M.W. and J.Y.; investigation, M.W. and Q.H.; resources, J.Y. and H.S.; data curation, M.W. and Q.H.; writing—original draft preparation, M.W., J.Y., Q.H., H.S., and F.S.; writing-review and editing, Q.H., H.S. and F.S.; visualization, M.W. and H.S.; supervision, Y.H., H.S. and F.S.; project administration, Y.H. and F.S.; funding acquisition, Y.H. All authors have read and agree to the published version of the manuscript.

Funding: This research was supported by the Science Technology Project Fund of the State Grid Corporation of China "Research and application of retail price policy under the mode of power sale side liberalization" (SGTYHT/17-JS-199), Fundamental Research Funds for the Central Universities (2019 QN078).

Acknowledgments: This paper mainly collects the initial data from the project. Some data have signed confidentiality agreement. Only the data used in the paper can be made public. Some data in the case study comes from the official website http://www.stats.gov.cn/.

Conflicts of Interest: The authors declare that they have no conflicts of interest.

\section{References}

1. The State Council of the People's Republic of China. Relative Policies on Deepening the Reform of Power Industry. Available online: httlp://www.gov.cn/zhengce/xxgkzl.html (accessed on 1 May 2019).

2. Zeng, M.; Yang, Y.; Wang, L.; Sun, J. The power industry reform in China 2015: Policies, evaluations and solutions. Renew. Sustain. Energy Rev. 2016, 57, 94-110. [CrossRef]

3. Ding, Y.; Hui, H.; Lin, Z.; Zheng, M.; Qu, X.; Cui, W. Design of business model and market framework oriented to active demand response of power demand side. Autom. Electr. Power Syst. 2017, 41, 2-9. (In Chinese)

4. Zhao, C.; Zhang, S.H. Game model of electricity retail market considering consumers'switching behaviors. Electr. Power Autom. Equip. 2020, 40, 162-168.

5. Cavanagh, R.; Levin, A. Rehabilitating retail electricity markets: Pitfalls and opportunities. In Future of Utilities Utilities of the Future; Elsevier: London, UK, 2016; pp. 175-192. 
6. Bae, M.; Kim, H.; Kim, E.; Chung, A.Y.; Kim, H.; Roh, J.H. Toward electricity retail competition: Survey and case study on technical infrastructure for advanced electricity market system. Appl. Energy 2014, 133, 252-273. [CrossRef]

7. Bai, Y.; Xie, L.; Zhong, H.; Xia, Q.; Chen, Q. Institutional design of Chinese retail electricity market reform and related suggestions. Autom. Electr. Power Syst. 2015, 39, 1-7. (In Chinese)

8. Zhang, X.; Xue, S.; Yang, S.; Tu, J.; Wei, Z.; Ma, L. International experience and lessons in power sales side market liberalization. Autom. Electr. Power Syst. 2016, 40, 1-8.

9. Types of Electric Plans, Public Utility Commission of Texas [EB/OL]. Available online: http://www.puc.texas. gov/consumer/facts/factsheets/elecfacts/Electricplans.pdf (accessed on 1 September 2018).

10. Zhang, X.P.; Li, J.; Fu, H. UK retail electricity market reform and challenges. Autom. Electr. Power Syst. 2016, 40, 10-16. (In Chinese) [CrossRef]

11. Hu, C.; Du, S.; Su, J.; Tong, G.; Wang, M. Preliminary research of trading approach and management modes of chinese electricity retail companies under new electricity market reform. Power Syst. Technol. 2016, 40, 3293-3299.

12. Wang, L.; Zhang, L.; Zhang, F.; Jin, D. Decision-making and Risk Assessment of Purchasing and Selling Business for Electricity Retailers. Autom. Electr. Power Syst. 2018, 42, 47-54.

13. Adomavicius, G.; Tuzhilin, A. Toward the next generation of recommender systems: A survey of the state-of-the-art and possible extensions. IEEE Trans. Knowl. Data Eng. 2005, 17, 734-749. [CrossRef]

14. Cui, L.Z.; Huang, W.Y.; Yan, Q.; Yu, F.R.; Wen, Z.K.; Lu, N. A novel context-aware recommendation algorithm with two-level SVD in social networks. Future Gener. Comput. Syst. 2018, 17, 1459-1470. [CrossRef]

15. Energy Made Easy. Available online: https://www.energymadeeasy.gov.au/offer-search (accessed on 15 December 2019).

16. iSelect. Available online: http://www.iselect.com.au/energy/ (accessed on 15 December 2019).

17. Power to Choose. Available online: http://www.powertochoose.org (accessed on 18 December 2019).

18. Check24. Available online: https://www.check24.de/strom-gas/ (accessed on 22 December 2019).

19. Silva, V.; Rodrigues, F.; Pinto, R.; Vale, Z. A data mining decision support tool to study electricity retail contracts. In Proceedings of the 2005 8th Congresso Luso-Espanhol de Energia, Marbella, Spain, 14-17 January 2005; pp. 1-7.

20. Zhang, Y.; Meng, K.; Dong, X.; Lai, M.; Yu, Z. Recommending electricity plans: A data-driven method. IEEE Int. Conf. Smart Grids Commun. 2016, 2, 668-673.

21. Luo, F.; Ranzi, G.; Wang, X.; Dong, Z.Y. Social information filtering-based electricity retail plan recommender system for smart grid end users. IEEE Trans. Ind. Inform. 2019, 10, 95-104. [CrossRef]

22. Zhang, Y.; Meng, K.; Kong, W.; Dong, Z.Y.; Qian, F. Bayesian hybrid collaborative filtering-based residential electricity plan recommender system. IEEE Trans. Ind. Inform. 2019, 15, 4731-4741. [CrossRef]

23. Yang, J.H.; Wang, H.B.; Gao, C.H.; Dai, Y.; Lv, Z.L. The application of social tagging based collaborative filtering personal recommender strategy in electricity market. Int. Conf. Comput. Inf. Syst. Ind. Appl. 2015, 18, 253-255.

24. Li, S.; Luo, F.; Yang, J.; Ranzi, G.; Wen, J. A personalized electricity tariff recommender system based on advanced metering infrastructure and collaborative filtering. Electr. Power Energy Syst. 2019, 113, 403-410. [CrossRef]

25. Jiménez-Bravo, D.M.; Pérez-Marcos, J.; De la Iglesia, D.H.; Villarrubia, G.; De Paz, J.F. Multi-agent recommendation system for electrical energy optimization and cost saving in smart homes. Energies 2019, 12, 1317. [CrossRef]

26. Aalami, H.; Moghaddam, M.; Yousefi, G. Evaluation of nonlinear models for time-based rates demand response programs. Int. J. Electr. Power Energy Syst. 2015, 65, 282-290. [CrossRef]

27. Zhang, Y.; Meng, K.; Kong, W.; Dong, Z.Y. Collaborative filtering-based electricity plan recommender system. IEEE Trans. Ind. Inform. 2019, 15, 1393-1404. [CrossRef]

28. Luo, X.; Zhou, M.; Xia, Y.; Zhu, Q. An efficient non-negative matrix-factorization-based approach to collaborative filtering for recommender systems. IEEE Trans. Ind. Inform. 2014, 10, 1273-1284.

29. Huang, B.H.; Dai, B.R. A weighted distance similarity model to improve the accuracy of collaborative recommender system. In Proceedings of the 2015 16th IEEE International Conference on Mobile Data Management, Pittsburgh, PA, USA, 15-18 June 2015; Volume 8, pp. 104-109. 
30. Kwon, H.J.; Hong, K.S. Personalized smart TV program recommender based on collaborative filtering and a novel similarity method. IEEE Trans. Consum. Electron. 2016, 57, 1416-1423. [CrossRef]

31. Guang, F.; He, Y.; Wen, L. Impacts of hybrid time-varying tariffs on residential electricity demand: The case of Zhejiang Province. Util. Policy 2019, 61, 1-9. [CrossRef]

32. Mikeli, A.; Apostolou, D.; Despotis, D. A multi-criteria recommendation method for interval scaled ratings. In Proceedings of the 2013 IEEE/WIC/ACM International Joint Conferences on Web Intelligence and Intelligent Agent Technologies, Atlanta, GA, USA, 17-20 November 2013; Volume 12, pp. 9-12.

33. Hdioud, F.; Frikh, B.; Ouhbi, B. Multi-criteria recommender systems based on multi-attribute decision making. In Proceedings of the 15th International Conference on Information Integration and Web-based Applications \& Services, Vienna, Austria, 2-4 December 2013; ACM: New York, NY, USA, 2013; pp. $203-211$.

34. Chulyadyo, R.; Leray, P. A personalized recommender system from probabilistic relational model and users' preferences. Procedia Comput. Sci. 2014, 35, 1063-1072. [CrossRef]

35. Khadgi, P.; Bai, L. A simulation study for residential electricity user behavior under dynamic variable pricing with demand charge. IISE Trans. 2018, 50, 699-710. [CrossRef]

(C) 2020 by the authors. Licensee MDPI, Basel, Switzerland. This article is an open access article distributed under the terms and conditions of the Creative Commons Attribution (CC BY) license (http://creativecommons.org/licenses/by/4.0/). 